\title{
Orders of automorphisms of smooth plane curves for the automorphism groups to be cyclic
}

Received: 22 August 2020 / Accepted: 6 March 2021 / Published online: 18 May 2021

(C) The Author(s) 2021

\begin{abstract}
For a fixed integer $d \geq 4$, the list of groups that appear as automorphism groups of smooth plane curves whose degree is $d$ is unknown, except for $d=4$ or 5. Harui showed a certain characteristic about structures of automorphism groups of smooth plane curves. Badr and Bars began to study for certain orders of automorphisms and try to obtain exact structures of automorphism groups of smooth plane curves. In this paper, based on the result of T. Harui, we extend Badr-Bars study for different and new cases, mainly for the cases of cyclic groups that appear as automorphism groups.
\end{abstract}

Mathematics Subject Classification $14 \mathrm{H} 50 \cdot 14 \mathrm{H} 37$

\section{Introduction}

In this paper, we work over $\mathbb{C}$. Let $C$ be a smooth plane curve of degree $d \geq 4$, and $\operatorname{Aut}(C)$ be the automorphism group of $C$. The group $\operatorname{Aut}(C)$ is a finite subgroup of the group PGL $(3, \mathbb{C})=\operatorname{Aut}\left(\mathbb{P}^{2}\right)[17]$. For $d=4$, Henn [11] and Kuribayashi-Komiya [14] independently give a list of precise automorphism groups that appear in a smooth plane curve of degree 4, see [1] for a survey of both results for quartics. For $d=5$, Badr and Bars give a precise list [2]. In [6], Harui provides a list of group structures that may be displayed as automorphism groups of smooth plane curves of degree $d \geq 4$. Part of his list are expressed using exact sequences of groups, and exact structures are unknown. Especially when the degree $d$ is fixed, his list does not clearly state group structures which are actually realized as automorphism groups of smooth plane curves of degree $d$.

For an automorphism $\sigma \in \operatorname{Aut}(C)$, we write the order of $\sigma$ as $\operatorname{ord}(\sigma)$. In this paper, we study the group structure of $\operatorname{Aut}(C)$ from ord $(\sigma)$. In [3], Badr and Bars shows that ord $(\sigma)$ divides one of $(d-2)(d-1)+1$, $(d-2) d,(d-1)^{2}$, and $(d-1) d$. In particular, when ord $(\sigma)$ is one of $(d-2)(d-1)+1,(d-2) d,(d-1)^{2}$, and $(d-1) d$, they show that the pair $C$ and $\operatorname{Aut}(C)$ are uniquely determined.

We fix $d \geq 4$, and let $\mathcal{G}_{d}$ be the set consisting of cyclic groups $\mathcal{C} y c_{m}$, dihedral groups $D_{m}$, the tetrahedral group $A_{4}$, the octahedral group $\mathcal{S}_{4}$, and the icosahedral group $A_{5}$ where $m \leq d-1$.

Let $F_{d}$ be the Fermat curve defined by $X^{d}+Y^{d}+Z^{d}=0$, and $K_{d}$ be the Klein curve defined by $Z X^{d-1}+X Y^{d-1}+Y Z^{d-1}=0$. The group structures of the automorphism groups $F_{d}$ and $K_{d}$ are known $[4,19]$.

In [3], Badr and Bars study the group structure of $\operatorname{Aut}(C)$ when ord $(\sigma)=k(d-2), k(d-1)$, or $k d$ for $k \geq 2$, and show the following theorem.

Theorem 1.1 (Please see [3] for details) Let $C$ be a smooth plane curve of degree $d \geq 4$, Aut $(C)$ be the automorphism group of $C, \sigma \in \operatorname{Aut}(C)$ be an automorphism, and $k \geq 2$.

T. Hayashi $(\bowtie)$

Faculty of Agriculture, Kindai University, Nakamaticho 3327-204, Nara, Nara 631-8505, Japan

E-mail: haya4taro@gmail.com 
1. If $\operatorname{ord}(\sigma)=k(d-1)$, then $\operatorname{Aut}(C)$ is a cyclic group.

2. If $\operatorname{ord}(\sigma)=k d$, then $\operatorname{Aut}(C))$ is a subgroup of $\operatorname{Aut}\left(F_{d}\right)$ or there is a normal subgroup $H$ of $\operatorname{Aut}(C)$ such that the quotient group $\operatorname{Aut}(C) / H$ is one of $\mathcal{G}_{d}$.

3. If $\operatorname{ord}(\sigma)=k(d-2), d>6$, and $d \neq 10$, then there is a normal cyclic group $H$ such that the quotient group $\operatorname{Aut}(C) / H$ is a dihedral group.

Our first main theorem is the following Theorem 1.2 which is a continuation work of Theorem 1.1 based on [6]. We write the greatest common divisor of positive integers $s$ and $t$ as $\operatorname{gcd}(s, t)$. Let $C$ be a smooth plane curve of degree $d \geq 4$, and $\sigma$ be the automorphism group of $C$ such that $\operatorname{ord}(\sigma) \geq 4$. Notice that if $\operatorname{gcd}(\operatorname{ord}(\sigma), d-1) \geq 2$, then ord $(\sigma)$ divides $(d-1) d$ or $(d-1)^{2}$, if $\operatorname{gcd}(\operatorname{ord}(\sigma), d) \geq 2$, then $\operatorname{ord}(\sigma)$ divides $(d-1) d$ or $(d-2) d$, and if $\operatorname{gcd}(\operatorname{ord}(\sigma), d-2) \geq 2$, then ord $(\sigma)$ divides $(d-2) d$.

Theorem 1.2 Let $C$ be a smooth plane curve of degree $d \geq 4$, and $\operatorname{Aut}(C)$ be the automorphism group of $C$. We assume that there is an automorphism $\sigma \in \operatorname{Aut}(C)$ such that $\operatorname{ord}(\sigma) \geq 8$.

1. If ord $(\sigma)$ divides $(d-1)^{2}$ but does not divide $d-1$, then Aut $(C)$ is a cyclic group.

2. If $\operatorname{gcd}(\operatorname{ord}(\sigma), d) \geq 2$, and $\operatorname{gcd}(\operatorname{ord}(\sigma), d-1) \geq 3$, then $\operatorname{Aut}(C)$ is a cyclic group or there is a normal subgroup $H$ of $\operatorname{Aut}(C)$ such that the quotient group $\operatorname{Aut}(C) / H$ is the tetrahedral group $A_{4}$. In particular, if $\operatorname{gcd}(\operatorname{ord}(\sigma), d) \geq 2$ and $\operatorname{gcd}(\operatorname{ord}(\sigma), d-1) \geq 4$, then $\operatorname{Aut}(C)$ is a cyclic group.

3. If $\operatorname{gcd}(\operatorname{ord}(\sigma), d-2) \geq 12$, then $\operatorname{Aut}(C)$ is a cyclic group, or there is a normal cyclic group $H$ of $\operatorname{Aut}(C)$ such that the quotient group $\operatorname{Aut}(C) / H$ is a dihedral group.

Notice that if the order ord $(\sigma)$ of $\sigma$ divides $(d-1)^{2}$ but not $d-1$, then ord $(\sigma)$ is not the product of different prime numbers, i.e. $\operatorname{ord}(\sigma) \neq p_{1} \times \cdots \times p_{r}$ where $p_{i}$ is a prime number, and $p_{i} \neq p_{j}$ for $1 \leq i<j \leq r$. In particular, $\operatorname{ord}(\sigma) \neq 2,3,5,6,7$. For $d \geq 6$, the (1) and (2) of Theorem 1.2 uses smaller orders than the (1) of Theorem 1.1 to give sufficient conditions for $\operatorname{Aut}(C)$ to be a cyclic group. Please see Examples 3.4 and 3.6. When $\operatorname{ord}(\sigma)$ is not a multiple of $d-2$, either case of the (3) of Theorem 1.2 is occurred. Please see Examples 3.9 and 3.10. For the case ord $(\sigma)$ divides $(d-2)(d-1)+1$, please see Theorem 3.11.

Among smooth plane curves of degree $d \geq 4$, characterizations of the Fermat curve $F_{d}$ using the structure or order of the automorphism group is known $[6,18]$. Our second main theorem is a characterization of the Fermat curve $F_{d}$ not by the order or structure of the automorphism group, but by the orders of two automorphisms and an automorphism obtained by their composition.

Theorem 1.3 Let $C$ be a smooth plane curve of degree $d \geq 4$, and Aut $(C)$ be the automorphism group of $C$. The curve $C$ has two automorphism $\sigma$ and $\varsigma$ such that $\operatorname{ord}(\sigma)=2 d, \operatorname{ord}(\varsigma)=3$, and $\operatorname{ord}\left(\varsigma \sigma^{2}\right)=3$ if and only if the curve $C$ is projectively equivalent to the Fermat curve $F_{d}$.

Section 2 is preliminary. We will explain the basic facts of the automorphism groups of smooth plane curves and the Galois point. To show Theorem 1.3, we will use the Galois point as a crucial step. Let $C$ be a smooth plane curve of degree $d \geq 4$, and $\sigma \in \operatorname{Aut}(C)$. Based on [3], we will explain the relationship between the order of $\sigma$ and the defining equation of $C$. To show Theorem 1.2, we will use the relationship. In Sect. 3, we will show Theorems 1.2 and 1.3.

\section{Preliminary}

Let $C$ be a smooth plane curve of degree $d \geq 4$, and $\operatorname{Aut}(C)$ be the automorphism group of the curve $C$. Recall that the group $\operatorname{Aut}(C)$ is a finite subgroup of the group PGL $(3, \mathbb{C})$.

Definition 2.1 For $d \geq 4$, the Fermat curve $F_{d}$ is a smooth plane curve defined by

$$
X^{d}+Y^{d}+Z^{d}=0
$$

and the Klein curve $K_{d}$ is a smooth plane curve defined by

$$
Z X^{d-1}+X Y^{d-1}+Y Z^{d-1}=0
$$


From here, we will explain the automorphism groups of the Klein curve $K_{d}$ and the Fermat curve $F_{d}$ which are the famous and important smooth plane curves $[4,19]$. First, we explain the automorphism group Aut $\left(F_{d}\right)$ of the Fermat curve $F_{d}$. Let $N_{F_{d}}$ be a subgroup of the group $\operatorname{Aut}\left(F_{d}\right)$ generated by

$$
\left(\begin{array}{ccc}
e_{d} & 0 & 0 \\
0 & 1 & 0 \\
0 & 0 & 1
\end{array}\right) \text { and }\left(\begin{array}{ccc}
1 & 0 & 0 \\
0 & e_{d} & 0 \\
0 & 0 & 1
\end{array}\right)
$$

Let $\mathcal{S}_{3}$ be the symmetric group of degree 3.

Theorem $2.2[6,19]$ Let $F_{d}$ be the Fermat curve.

1. The group $N_{F_{d}}$ is a normal subgroup of $\operatorname{Aut}\left(F_{d}\right)$, and $\operatorname{Aut}\left(F_{d}\right)$ is generated by

$$
\left(\begin{array}{ccc}
e_{d} & 0 & 0 \\
0 & 1 & 0 \\
0 & 0 & 1
\end{array}\right),\left(\begin{array}{ccc}
1 & 0 & 0 \\
0 & e_{d} & 0 \\
0 & 0 & 1
\end{array}\right),\left(\begin{array}{lll}
0 & 1 & 0 \\
1 & 0 & 0 \\
0 & 0 & 1
\end{array}\right) \text {, and }\left(\begin{array}{lll}
1 & 0 & 0 \\
0 & 0 & 1 \\
0 & 1 & 0
\end{array}\right)
$$

2. There is a split short exact sequence of groups:

$$
1 \rightarrow N_{F_{d}} \rightarrow \operatorname{Aut}\left(F_{d}\right) \rightarrow \mathcal{S}_{3} \rightarrow 1 .
$$

Notice that $\left\langle\left(\begin{array}{lll}0 & 1 & 0 \\ 1 & 0 & 0 \\ 0 & 0 & 1\end{array}\right),\left(\begin{array}{lll}1 & 0 & 0 \\ 0 & 0 & 1 \\ 0 & 1 & 0\end{array}\right)\right) \cong \mathcal{S}_{3}$.

Corollary 2.3 Let $F_{d}$ be the Fermat curve for $d \geq 4$. The order of an automorphism $\sigma$ of $F_{d}$ is 3 or divides $2 d$.

Proof Let $\sigma$ be an automorphism of $F_{d}$. Let $q: \operatorname{Aut}\left(F_{d}\right) \rightarrow \operatorname{Aut}\left(F_{d}\right) / N_{F_{d}}$ be the quotient morphism. Since the quotient group $\operatorname{Aut}\left(F_{d}\right) / N_{F_{d}}$ is $\mathcal{S}_{3}$, the order of $q(\sigma)$ is 2 or 3 as an element of $\operatorname{Aut}\left(F_{d}\right) / N_{F_{d}}$. If the order of $q(\sigma)$ is 2 , then $\sigma^{2} \in N_{F_{d}}$, and hence the order of $\sigma$ divides $2 d$. We assume that the order of $q(\sigma)$ is 3 . By Theorem 2.2, $\sigma$ is defined by a matrix $A=\left(\begin{array}{lll}0 & a & 0 \\ 0 & 0 & b \\ c & 0 & 0\end{array}\right)$. Since $A^{3}=\left(\begin{array}{ccc}a b c & a & 0 \\ 0 & a b c & 0 \\ 0 & 0 & a b c\end{array}\right), \sigma^{3}=\mathrm{id}_{F_{d}}$. Then the order of $\sigma$ is 3 .

The characterization of the Fermat curve $F_{d}$ using the order of the automorphism group is known as follows.

Theorem 2.4 [18] Let $C$ be a smooth plane curve of degree $d \geq 4$, and $\operatorname{Aut}(C)$ be the automorphism group of $C$. If the order of $\operatorname{Aut}(C)$ is $6 d^{2}$, then $C$ is projectively equivalent to the Fermat curve $F_{d}$.

We explain the automorphism group $\operatorname{Aut}\left(K_{d}\right)$ of the Klein curve $K_{d}$. Let $M_{K_{d}}$ be a subgroup of $\operatorname{Aut}\left(K_{d}\right)$ generated by

$$
\left(\begin{array}{ccc}
e_{d^{2}}-3 d+3 & 0 & 0 \\
0 & e_{d^{2}-3 d+3}^{d-3} & 0 \\
0 & 0 & 1
\end{array}\right)
$$

Theorem $2.5[4,6]$ Let $K_{d}$ be the Klein curve defined by $Z X^{d-1}+X Y^{d-1}+Y Z^{d-1}=0$.

1. If $d=4$, then the group $\operatorname{Aut}\left(K_{4}\right)$ is called the Klein group $\operatorname{PSL}\left(2, \mathbb{F}_{7}\right)$.

2. If $d \geq 5$, then the group $M_{K_{d}}$ is a normal subgroup of $\operatorname{Aut}\left(K_{d}\right)$, and the group $\operatorname{Aut}\left(K_{d}\right)$ is generated by

$$
\left(\begin{array}{ccc}
e_{d^{2}}-3 d+3 & 0 & 0 \\
0 & e_{d^{2}-3 d+3}^{d-3} & 0 \\
0 & 0 & 1
\end{array}\right) \text { and }\left(\begin{array}{lll}
0 & 1 & 0 \\
0 & 0 & 1 \\
1 & 0 & 0
\end{array}\right)
$$

and there exists a split short exact sequence of groups:

$$
1 \rightarrow M_{K_{d}} \rightarrow \operatorname{Aut}\left(K_{d}\right) \rightarrow \mathcal{C} y c_{3} \rightarrow 1
$$


Corollary 2.6 Let $K_{d}$ be the Klein curve for $d \geq 5$. The order of an automorphism $\sigma$ of $K_{d}$ is 3 or divides $(d-2)(d-1)+1$.

Proof Let $\sigma$ be an automorphism of $K_{d}$. If $\sigma \in M_{K_{d}}$, then ord $(\sigma)$ divides $(d-2)(d-1)+1$. We assume that $\sigma \notin M_{K_{d}}$. By Theorem 2.5, $\sigma$ is defined by a matrix of the form $\left(\begin{array}{lll}0 & a & 0 \\ 0 & 0 & b \\ c & 0 & 0\end{array}\right)$. Then $\operatorname{ord}(\sigma)$ is 3 .

The characterization of the Klein curve $K_{d}$ using the order of an automorphism is known as follows.

Theorem $2.7[3,6]$ Let $C$ be a smooth plane curve of degree $d \geq 4$. If $C$ has an automorphsim of order $(d-2)(d-1)+1$, then $C$ is projectively equivalent to the Klein curve $K_{d}$.

Definition 2.8 [6] For a monomial $X^{i} Y^{j} Z^{k}$, let

$$
\operatorname{ex}\left(X^{i} Y^{j} Z^{k}\right):=\max \{i, j, k\} .
$$

For a homogeneous polynomial $F:=\sum_{i+j+k=d} a_{i, j, k} X^{i} Y^{j} Z^{k}$ of degree $d$, we set $l:=\max \left\{\operatorname{ex}\left(X^{i} Y^{j} Z^{k}\right)\right.$ : $\left.a_{i, j, k} \neq 0\right\}$. The core of $F$ is defined as

$$
\sum_{\operatorname{ex}\left(X^{i} Y^{j} Z^{k}\right)=l} a_{i, j, k} X^{i} Y^{j} Z^{k}
$$

Definition 2.9 [6] Let $C_{0}$ be a smooth plane curve of degree $d \geq 4$. A pair $(C, G)$ of a smooth plane curve $C$ and a subgroup $G$ of the group $\operatorname{Aut}(C)$ is said to be a descendant of $C_{0}$ if $C$ is defined by a homogeneous polynomial whose core is a defining polynomial of $C_{0}$, and $G$ is a subgroup of the group $\operatorname{Aut}\left(C_{0}\right)$ as a subgroup of the group PGL $(3, \mathbb{C})$ under a suitable coordinate system.

For a positive integer $m \geq 2$, let $e_{m}$ be a primitive $m$-th root of unity.

Definition 2.10 Let $\operatorname{PBD}(2,1)$ be the subgroup of $\operatorname{PGL}(3, \mathbb{C})$ that consists of all elements representable by a matrix of the form $\left(\begin{array}{ccc}a_{11} & a_{12} & 0 \\ a_{21} & a_{22} & 0 \\ 0 & 0 & 1\end{array}\right)$, and $H_{l}$ be a cyclic group of order $l$ generated by $\left(\begin{array}{ccc}e_{l} & 0 & 0 \\ 0 & e_{l} & 0 \\ 0 & 0 & 1\end{array}\right)$ for $l \geq 1$.

Notice that the group $H_{l}$ is contained in the center of $\operatorname{PBD}(2,1)$. Harui provides a list of group structures that may be displayed as automorphism groups of smooth plane curves as follows.

Theorem 2.11 [6] Let $C$ be a smooth plane curve of degree $d \geq 4$, Aut $(C)$ be the automorphism group of $C$, and $G$ a subgroup of $\operatorname{Aut}(C)$. Then one of the following holds.

(a-i) The group $G$ is a cyclic group.

(a-ii) The group $G$ fixes a point not lying on the curve $C$, by replacing the local coordinate system if necessary, $G \subset \operatorname{PBD}(2,1)$ and $H_{l} \subset G$ for some $l$ such that l divides $d, H_{l}$ is contained in the center of $G$, and the quotient group $G / H_{l}$ is one of $\mathcal{G}_{d}$. In addition, if $G / H_{l} \cong D_{m}$ and $H_{l}$ is non-trivial, then $m$ divides $d-2$.

(b-i) The pair $(C, G)$ is a descendant of the Fermat curve $F_{d}$.

(b-ii) The pair $(C, G)$ is a descendant of the Klein curve $K_{d}$.

(c) The group $G$ is primitive and conjugate to the icosahedral group $A_{5}$, the Klein group $\operatorname{PSL}\left(2, \mathbb{F}_{7}\right)$, the alternating group $A_{6}$, the Hessian group $H_{216}$ of order 216, or its subgroup of order 36 or 72.

The order of an element of the groups $A_{5}, \operatorname{PSL}\left(2, \mathbb{F}_{7}\right), A_{6}$, and $H_{216}$ is 7 or less. Therefore, if the automorphism group Aut $(C)$ of a smooth plane curve $C$ of degree $d \geq 4$ has an element of order 8 or more, the pair $(C, \operatorname{Aut}(C))$ is not one of the case $(c)$ of Theorem 2.11.

From here, based on [3], we will explain the relationship between the order of an automorphism and the defining equation of a smooth plane curve (see also [8]). Let $C$ be a smooth plane curve of degree $d \geq 4$, and $\sigma$ be an automorphism of $C$. We set Fix $(\sigma):=\{x \in C \mid g(x)=x\}$. By replacing the local coordinate system, we may assume that $\sigma$ is defined by a diagonal matrix $A:=\left(\begin{array}{ccc}\alpha & 0 & 0 \\ 0 & \beta & 0 \\ 0 & 0 & 1\end{array}\right)$. Let

$$
n(\sigma):=\sharp|\operatorname{Fix}(\sigma) \cap\{[1: 0: 0],[0: 1: 0],[0: 0: 1]\}| .
$$


Table 1 Cyclic groups of smooth plane curves of degree $d \geq 4$

\begin{tabular}{|c|c|c|c|c|}
\hline No. & $n(\sigma)$ & $F_{*}$ & $A$ & $\operatorname{ord}(\sigma)$ divides \\
\hline 1 & 0 & $X^{d}+Y^{d}+Z^{d}$ & $\left(\begin{array}{ccc}e_{l}^{s} & 0 & 0 \\
0 & e_{l}^{t} & 0 \\
0 & 0 & 1\end{array}\right)$ & $d$ \\
\hline 2 & 1 & $(Y+Z) X^{d-1}+Y^{d}+Z^{d}$ & $\begin{array}{l}\left(\begin{array}{lll}e_{l} & 0 & 0 \\
0 & 1 & 0 \\
0 & 0 & 1\end{array}\right) \\
\left(\begin{array}{lll}e_{l} & 0 & 0\end{array}\right)\end{array}$ & $d-1$ \\
\hline 3 & 1 & $Y X^{d-1}+Y^{d}+Z^{d}$ & 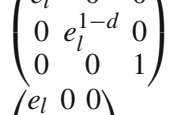 & $(d-1) d$ \\
\hline 4 & 2 & $(Y+Z) X^{d-1}+Z Y^{d-1}+Z^{d}$ & $\left(\begin{array}{lll}0 & 1 & 0 \\
0 & 0 & 1\end{array}\right)$ & $d-1$ \\
\hline 5 & 2 & $Z X^{d-1}+Z Y^{d-1}+Z^{d}$ & $\left(\begin{array}{lll}l & & 0 \\
0 & e_{l}^{t} & 0 \\
0 & 0 & 1\end{array}\right)$ & $d-1$ \\
\hline 6 & 2 & $Z X^{d-1}+X Y^{d-1}+Z^{d}$ & $\left(\begin{array}{ccc}e_{l}^{1-d} & 0 & 0 \\
0 & e_{l} & 0 \\
0 & 0 & 1\end{array}\right)$ & $(d-1)^{2}$ \\
\hline 7 & 2 & $Y X^{d-1}+X Y^{d-1}+Z^{d}$ & $\left.\begin{array}{ccc}e_{l} & 0 & 0 \\
0 & e_{l}^{1-d} & 0 \\
0 & 0 & 1\end{array}\right)$ & $(d-2) d$ \\
\hline 8 & 3 & $(Y+Z) X^{d-1}+Z Y^{d-1}+Y Z^{d-1}$ & $\left(\begin{array}{lll}0 & 1 & 0 \\
0 & 0 & 1\end{array}\right)$ & $d-1$ \\
\hline 9 & 3 & $Z X^{d-1}+Z Y^{d-1}+Y Z^{d-1}$ & $\begin{array}{l}\left(\begin{array}{lll}e_{l} & 0 & 0 \\
0 & 1 & 0 \\
0 & 0 & 1\end{array}\right) \\
\left(\begin{array}{lll}e_{l} & 0 & 0\end{array}\right)\end{array}$ & $d-1$ \\
\hline 10 & 3 & $Z X^{d-1}+X Y^{d-1}+Y Z^{d-1}$ & 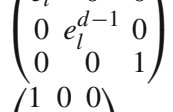 & $d^{2}-3 d+3$ \\
\hline 11 & 3 & $Z X^{d-1}+X Y^{d-1}+X Z^{d-1}$ & $\left(\begin{array}{lll}0 & e_{l} & 0 \\
0 & 0 & 1\end{array}\right)$ & $d-1$ \\
\hline
\end{tabular}

Notice that $n(\sigma)=0,1,2$, or 3 . Since $\sigma$ is defined by a diagonal matrix, $n(\sigma)=C \cap\{[1: 0: 0],[0: 1$ : $0]$, $[0: 0: 1]\}$. We write the defining equation $F(X, Y, Z)$ of the curve $C$ as follows.

$$
F(X, Y, Z)=\sum_{0 \leq i, j \leq d} a_{i j} X^{i} Y^{j} Z^{d-i-j}
$$

For $F(X, Y, Z)=\sum_{0 \leq i, j \leq d} a_{i j} X^{i} Y^{j} Z^{d-i-j}$, we set

$$
F_{*}:=\sum_{\min \{i, j, d-i-j\} \geq d-1} a_{i j} X^{i} Y^{j} Z^{d-i-j} .
$$

The following theorem holds.

Theorem 2.12 [3] By replacing the local coordinate system, the number $n(\sigma)$, the polynomial $F_{*}$, the diagonal matrix $A$, and the order $\operatorname{ord}(\sigma)$ are one of Table 1. In particular, $\operatorname{ord}(\sigma)$ divides one of $(d-2)(d-1)+1$, $(d-2) d,(d-1)^{2}$, and $(d-1) d$.

Theorem 2.12 determines the orders of automorphisms, that is, the cyclic groups acting on smooth plane curves of degree $d \geq 4$. In [10], the structures of abelian groups which are not cyclic groups and act on smooth plane curves of degree $d \geq 4$ are determined. Besides, in [9], the structures of groups which act freely on smooth plane curves of degree $d \geq 4$ are determined.

Definition 2.13 Let $C_{(d-1) d}$ be a smooth plane curve defined by

$$
Y X^{d-1}+Y^{d}+Z^{d} .
$$


Let $C_{(d-1)^{2}}$ be a smooth plane curve defined by

$$
Z X^{d-1}+X Y^{d-1}+Z^{d}=0 .
$$

Let $C_{(d-2) d}$ be a smooth plane curve defined by

$$
Y X^{d-1}+X Y^{d-1}+Z^{d}=0 .
$$

The automorphism groups of the curves $C_{(d-1) d}, C_{(d-1)^{2}}$, and $C_{(d-2) d}$ are as follows.

Theorem $2.14[3,6]$ We have the following.

1. For $d \geq 5$, the automorphism group Aut $\left(C_{(d-1) d}\right)$ is a cyclic group of order $(d-1) d$ which is generated by an automorphism $\sigma:=\left(\begin{array}{ccc}e_{(d-1) d} & 0 & 0 \\ 0 & e_{(d-1) d}^{1-d} & 0 \\ 0 & 0 & 1\end{array}\right)$.

2. The automorphism group Aut $\left(C_{(d-1)^{2}}\right)$ is a cyclic group of order $(d-1)^{2}$ which is generated by an automorphism $\sigma:=\left(\begin{array}{ccc}e_{(d-1)^{2}}^{1-d} & 0 & 0 \\ 0 & e_{(d-1)^{2}} & 0 \\ 0 & 0 & 1\end{array}\right)$.

3. The curve $C_{(d-2) d}$ has an automorphism $\sigma:=\left(\begin{array}{ccc}e_{(d-2) d} & 0 & 0 \\ 0 & e_{(d-2) d}^{1-d} & 0 \\ 0 & 0 & 1\end{array}\right)$ of order $(d-2) d$.

4. The automorphism group $\operatorname{Aut}\left(C_{(d-2) d}\right)$ is generated by two automorphism $\sigma$ and $\varsigma:=\left(\begin{array}{lll}0 & 1 & 0 \\ 1 & 0 & 0 \\ 0 & 0 & 1\end{array}\right)$. In particular, the cyclic group $\left\langle\sigma^{d-2}\right\rangle$ is a normal subgroup of $\operatorname{Aut}\left(C_{(d-2) d}\right)$ such that the quotient group $\operatorname{Aut}\left(C_{(d-2) d}\right) /\left\langle\sigma^{d-2}\right\rangle$ is $D_{d-2}$.

The characterization of the curves $C_{(d-1) d}, C_{(d-1)^{2}}$, and $C_{(d-2) d}$ using the order of an automorphism is known as follows.

Theorem 2.15 [3] Let $C$ be a smooth plane curve of degree $d \geq 4$.

1. If $C$ has an automorphsim of order $(d-1) d$, then $C$ is projectively equivalent to the curve $C_{(d-1) d}$.

2. If $C$ has an automorphsim of order $(d-1)^{2}$, then $C$ is projectively equivalent to the curve $C_{(d-1)^{2}}$.

3. If $C$ has an automorphsim of order $(d-2) d$, then $C$ is projectively equivalent to the curve $C_{(d-2) d}$.

Let $C$ be a smooth plane curve of degree $d \geq 4, \operatorname{Aut}(C)$ be the automorphism group of $C$, and $\sigma$ be an automorphism of $C$. Recall that ord $(\sigma)$ divides $(d-2)(d-1)+1,(d-2) d,(d-1)^{2}$, or $(d-1) d$. Theorems 2.7 and 2.15 show that if ord $(\sigma)=(d-2)(d-1)+1,(d-2) d,(d-1)^{2}$, or $(d-1) d$, then $C$ is uniquely determined, and hence the structure of the group $\operatorname{Aut}(C)$ is also uniquely determined.

From here, we will explain the Galois point. Let $\mathbb{P}^{1}$ be the projective line. The gonality of a curve $C$ is $\operatorname{gon}(C):=\min \left\{\operatorname{deg} \varphi \mid \varphi: C \rightarrow \mathbb{P}^{1}\right\}$. If $C$ is a smooth plane curve of degree $d \geq 3$, then $\operatorname{gon}(C)=d-1$, and the gonality of $C$ is given by a projection $\pi_{p}: C \rightarrow \mathbb{P}^{1}$ at a point $p \in C$ [17].

Definition 2.16 $[16,20]$ Let $C$ be an irreducible plane curve of degree $d \geq 4$. A point $p \in \mathbb{P}^{1}$ is called a Galois point of $C$ if the projection $\pi_{p}: C \rightarrow \mathbb{P}^{1}$ is a Galois cover. Moreover, if $p \in C$ (resp. $p \in \mathbb{P}^{2} \backslash C$ ), then the point is said to be an inner (resp. outer) Galois point.

Theorem 2.17 $[16,20]$ Let $C$ is a smooth plane curve of degree $d \geq 4$. If $p \in \mathbb{P}^{2}$ is an inner (resp. outer) Galois point of $C$, then the Galois group of the Galois cover $\pi_{p}: C \rightarrow \mathbb{P}^{1}$ is a cyclic group of order $d-1$ (resp. d) which is generated by an automorphism $\sigma$ of $C$.

Definition 2.18 Let $C$ be an irreducible plane curve of degree $d \geq 4$. We denote the number of inner (resp. outer) Galois points of $C$ by $\delta(C)$ (resp. $\delta^{\prime}(C)$ ).

Theorem 2.19 [16,20]) Let $C$ is a smooth plane curve of degree $d \geq 4$. We have the following.

1. The number $\delta(C)=0,1$, or 4 , and the number $\delta^{\prime}(C)=0,1$, or 3 . 
2. If $\delta(C) \geq 1$ and $\delta^{\prime}(C) \geq 1$, then $\delta(C)=4$ and $\delta^{\prime}(C)=1$.

3. The number $\delta(C)=4$ if and only if $C$ is projectively equivalent to the curve $X Z^{3}+X^{4}+Y^{4}=0$.

4. The number $\delta^{\prime}(C)=3$ if and only if $C$ is projectively equivalent to the Fermat curve $F_{d}$.

5. If $\delta(C)=1$, then $\operatorname{Aut}(C)$ is a cyclic group.

6. If $\delta^{\prime}(C)=1$, then the pair $(C, \operatorname{Aut}(C))$ is the case (a-ii) of Theorem 2.11. In particular, let $G$ be the Galois group of the Galois cover $\pi_{p}: C \rightarrow \mathbb{P}^{1}$ where $p \in \mathbb{P}^{2}$ is the Galois point of $C$. The quotient group $\operatorname{Aut}(C) / G$ is one of $\mathcal{G}_{d}$.

Theorem 2.20 $[3,10]$ Let $C$ be a smooth plane curve in of degree $d \geq 4$, and $\sigma$ be an automorphism of $C$.

1. If $\operatorname{ord}(g)=d-1$, then $\sharp|\operatorname{Fix}(g)| \neq 2$ if and only if $C$ has an inner Galois point $p$, and the group $\langle g\rangle$ is the Galois group of the Galois cover $\pi_{p}: C \rightarrow \mathbb{P}^{1}$.

2. If $\operatorname{ord}(\sigma)=k(d-1)$ for $k \geq 2$, then $C$ has an inner Galois point $p$, and the group $\left\langle\sigma^{k}\right\rangle$ is the Galois group of the Galois cover $\pi_{p}: C \rightarrow \mathbb{P}^{1}$.

3. If $\operatorname{ord}(g)=d$, then $\sharp|\operatorname{Fix}(g)| \neq 0$ if and only if $C$ has an outer Galois point $p$, and the group $\langle g\rangle$ is the Galois group of the Galois cover $\pi_{p}: C \rightarrow \mathbb{P}^{1}$.

4. If $\operatorname{ord}(\sigma)=k d$ for $k \geq 2$, then $C$ has an outer Galois point $p$, and the group $\left\langle\sigma^{k}\right\rangle$ is the Galois group of the Galois cover $\pi_{p}: C \rightarrow \mathbb{P}^{1}$.

Other characterizations of smooth plane curves with Galois points are known [12,13].

Recall that for $d \geq 4, \mathcal{G}_{d}$ is a set consisting of cyclic groups $\mathcal{C} y c_{m}$, dihedral groups $D_{m}$, the tetrahedral group $A_{4}$, the octahedral group $\mathcal{S}_{4}$, and the icosahedral group $A_{5}$ where $m \leq d-1$. By Theorems 2.19 and 2.20 , we have the following.

Theorem 2.21 [3] Let $C$ be a smooth plane curve of degree d, and Aut $(C)$ be the automorphism group of $C$. We take an automorphism $\sigma \in \operatorname{Aut}(C)$.

1. If $d \geq 5$, and $\operatorname{ord}(\sigma)=k(d-1)$ for $k \geq 2$, then $\operatorname{Aut}(C)$ is a cyclic group.

2. If $\operatorname{ord}(\sigma)=k d$ for $k \geq 2$, then the quotient group $\operatorname{Aut}(C) /\left\langle\sigma^{k}\right\rangle$ is one of $\mathcal{G}_{d}$, or $\operatorname{Aut}(C) \cong \operatorname{Aut}\left(F_{d}\right)$ as a group.

There are studies on the automorphism groups of plane curves using Galois points [3,5,7,15]. In [3], for the case where ord $(\sigma)=k(d-2), k(d-1)$, or $k d$ for $k \geq 2$, the structure of $\operatorname{Aut}(C)$ is classified in detail.

\section{Proof of Theorems 1.2, and 1.3}

First, we will show the case (1) of Theorem 1.2. We will establish Lemma 3.1 and 3.2 in preparation for that. Recall that PSL $\left(2, \mathbb{F}_{7}\right)$ is the Klein group, $A_{6}$ is the alternating group, and $H_{216}$ is the Hessian group of order 216.

Lemma 3.1 Let $C$ be a smooth plane curve of degree $d \geq 4$, and $\operatorname{Aut}(C)$ be the automorphism group of $C$. If there is an automorphism $\sigma \in \operatorname{Aut}(C)$ such that $\operatorname{ord}(\sigma) \geq 8$, and $\operatorname{ord}(\sigma)$ divides $(d-1)^{2}$ but does not divide $d-1$, then the pair $(C, \operatorname{Aut}(C))$ is the case $(a-i)$ or $(a-i i)$ of Theorem 2.11.

Proof Let $\sigma \in \operatorname{Aut}(C)$ be an automorphism such that $\operatorname{ord}(\sigma) \geq 8$, and $\operatorname{ord}(\sigma)$ divides $(d-1)^{2}$ but does not divide $d-1$. Since orders of the elements of the groups $A_{5}, \operatorname{PSL}\left(2, \mathbb{F}_{7}\right), A_{6}$, and $H_{216}$ is 7 or less, we get that the pair $(C$, Aut $(C))$ is not the case $(c)$ of Theorem 2.11. Since ord $(\sigma) \geq 5$, and ord $(\sigma)$ divides $(d-1)^{2}$ but does not divide $d-1$ Corollary 2.6, we get that the pair $(C$, Aut $(C))$ is not the case $(b-i i)$ of Theorem 2.11. Same, since Corollary 2.3, the pair $(C, \operatorname{Aut}(C))$ is not the case $(b-i)$ of Theorem 2.11.

Lemma 3.2 Let $C$ be a smooth plane curve of degree $d \geq 4$, and $\operatorname{Aut}(C)$ be the automorphism group of $C$. We assume that the pair $(C$, Aut $(C))$ is the case (a-ii) of Theorem 2.11. We take an automorphism $\sigma$. Let $F$ be the defining equation of $C$, and $A$ be a matrix defining $\sigma$. By replacing the local coordinate system, we get that $F_{*}$ and $A$ are one of Table $1,[0: 0: 1] \notin C$, and $\operatorname{Aut}(C) \subset \operatorname{PBD}(2,1)$.

Proof Since the pair $(C, \operatorname{Aut}(C))$ is the case $(a-i i)$ of Theorem 2.11, by replacing the local coordinate system, we get that $[0: 0: 1] \notin C$, and $\operatorname{Aut}(C) \subset \operatorname{PBD}(2,1)$. Let $A=\left(\begin{array}{ccc}a_{11} & a_{12} & 0 \\ a_{21} & a_{22} & 0 \\ 0 & 0 & 1\end{array}\right)$ be a defining matrix of $\sigma$. From 
the form of the matrix $A$, there is a matrix $B=\left(\begin{array}{ccc}b_{11} & b_{12} & 0 \\ b_{21} & b_{22} & 0 \\ 0 & 0 & 1\end{array}\right)$ such that $B^{-1} A B$ is a diagonal matrix. Since $B([0: 0: 1])=[0: 0: 1]$, and $B^{-1}(\operatorname{PBD}(2,1)) B=\operatorname{PBD}(2,1)$. Therefore, by replacing the local coordinate system, we get that $\sigma$ is defined by a diagonal matrix, $[0: 0: 1] \notin C$, and $\operatorname{Aut}(C) \subset \operatorname{PBD}(2,1)$.

Let $F$ be the defining equation of $C$. Since the automorphism $\sigma$ is defined by a diagonal matrix, and Theorem 2.12, by multiplying $X, Y$, and $Z$ by a constant or permuting $X$ and $Y$, we get that the pair $(C, \sigma)$ is one of Table 1, i.e. $F_{*}$ and $A$ are one of Table 1 . Multiplying $X, Y$, and $Z$ by a constant and permuting $X$ and $Y$ do not change the situation where $[0: 0: 1] \notin C$ and $\operatorname{Aut}(C) \subset \operatorname{PBD}(2,1)$. Therefore, we get this lemma. $\square$

The following Theorem 3.3 corresponds to the (1) of Theorem 1.2.

Theorem 3.3 Let $C$ be a smooth plane curve of degree $d \geq 4$, and $\operatorname{Aut}(C)$ be the automorphism group of $C$. If there is an automorphism $\sigma \in \operatorname{Aut}(C)$ such that $\operatorname{ord}(\sigma) \geq 8$, and $\operatorname{ord}(\sigma)$ divides $(d-1)^{2}$ but does not divide $d-1$, then the group $\operatorname{Aut}(C)$ is a cyclic group.

Proof Let $\sigma \in \operatorname{Aut}(C)$ be an automorphism such that $\operatorname{ord}(\sigma) \geq 5$, and $\operatorname{ord}(\sigma)$ divides $(d-1)^{2}$ but does not divide $d-1$. By Lemma 3.1, the pair $(C$, Aut $(C))$ is the case $(a-i)$ or $(a-i i)$ of Theorem 2.11.

We assume that the pair $(C, \operatorname{Aut}(C))$ is the case $(a-i i)$ of Theorem 2.11. From here, we will show that the group $\operatorname{Aut}(C)$ is a cyclic group. By the case $(a-i i)$ of Theorem 2.11, there is a normal cyclic subgroup $H$ of Aut $(C)$ such that $H$ is contained in the center of $\operatorname{Aut}(C)$, the order of $H$ divides $d$, and the quotient group $\operatorname{Aut}(C) / H$ is one of $\mathcal{G}_{d}$. Since $\operatorname{gcd}\left(d,(d-1)^{2}\right)=1$, the group $\langle\sigma\rangle \cap H=\left\{\operatorname{id}_{C}\right\}$. Since $H$ is contained in the center of $\operatorname{Aut}(C),\langle\sigma, H\rangle=\langle\sigma\rangle \oplus H$. Since $\operatorname{gcd}\left(d,(d-1)^{2}\right)=1$, the group $\langle\sigma, H\rangle$ is a cyclic group. Let $\varsigma$ be a generator of $\langle\sigma, H\rangle$, and $v:=\sharp|H|$. Then $\operatorname{ord}(\varsigma)=v \times \operatorname{ord}(\sigma)$. By Theorem 2.12, we get that $v=1$, and hence the subgroup $H$ is trivial. Therefore, the group $\operatorname{Aut}(C)$ is one of $\mathcal{G}_{d}$. Then the cyclic group $\langle\sigma\rangle$ is isomorphic to a subgroup of the groups $\mathcal{C} y c_{m}, D_{m}, A_{4}, \mathcal{S}_{4}$, or $A_{5}$ where $m \leq d-1$. Since $\operatorname{ord}(\sigma) \geq 8$, and orders of the elements of the groups $A_{4}, \mathcal{S}_{4}$, and $A_{5}$ is 5 or less, we get that the group $\operatorname{Aut}(C)$ is not one of the groups $A_{4}, \mathcal{S}_{4}$, and $A_{5}$.

We assume that the group $\operatorname{Aut}(C)$ is a dihedral group $D_{m}$ where $m \leq d-1$. Since $\operatorname{ord}(\sigma) \geq 8$, there is an automorphism $\tau \in \operatorname{Aut}(C)$ of order 2 such that $\tau \sigma \tau=\sigma^{-1}$. Since Lemma 3.2, $\operatorname{ord}(\sigma)$, and Theorem 2.12, by replacing the local coordinate system, we get that $[0: 0: 1] \notin C$, and $\operatorname{Aut}(C) \subset \operatorname{PBD}(2,1)$, and the pair $(C, \sigma)$ is No. 6 of Table 1, i.e. $C$ and $\sigma$ are defined as follows:

$$
Z X^{d-1}+X Y^{d-1}+Z^{d}+\sum_{i=0}^{d-2} H_{d-i}(Y, Z) X^{i}=0 \text { and }\left(\begin{array}{ccc}
e_{l}^{1-d} & 0 & 0 \\
0 & e_{l} & 0 \\
0 & 0 & 1
\end{array}\right)
$$

where $l:=\operatorname{ord}(g), H_{d-i}(Y, Z)$ is a homogeneous polynomial of degree $d-i$ for $0 \leq i \leq d-2$ such that $H_{d-1}(Y, Z)$ does not have a $Y^{d-1}$ term, and $H_{d}(Y, Z)$ does not have $Y^{d}, Z^{d}$, and $Y^{d-1} Z$ terms.

Since ord $(\sigma)$ divides $(d-1)^{2}$, and $\operatorname{ord}(\sigma)$ does not divide $d-1$, we get that $\operatorname{Fix}(\sigma)$ is $\{[1: 0: 0],[0: 1: 0]\}$. Recall that the automorphism $\tau \in \operatorname{Aut}(C)$ is defined by a matrix of the form $\left(\begin{array}{ccc}c_{11} & c_{12} & 0 \\ c_{21} & c_{22} & 0 \\ 0 & 0 & 1\end{array}\right)$. Since $\tau \sigma \tau=\sigma^{-1}$ and $\operatorname{ord}(\tau)=2$, we get that $\tau(\operatorname{Fix}(\sigma))=\operatorname{Fix}(\sigma)$. Since $\operatorname{Fix}(\sigma)=\{[1: 0: 0],[0: 1: 0]\}$, we get that $\tau(\{[1: 0: 0],[0: 1: 0]\})=\{[1: 0: 0],[0: 1: 0]\}$. We assume that $\tau([1: 0: 0])=[1: 0: 0]$ or $\tau([0: 1: 0])=[0: 1: 0]$. Then $\operatorname{Fix}(\tau) \cap \operatorname{Fix}(\sigma) \neq \emptyset$. Since $C$ is smooth, the subgroup $\langle\tau, \sigma\rangle$ of $\operatorname{Aut}(C)$ is a cyclic group. This contradicts $\tau \sigma \tau=\sigma^{-1}$ and $\operatorname{ord}(\sigma) \geq 8$. Then $\tau([1: 0: 0])=[0: 1: 0]$ and $\tau([0: 1: 0])=[1: 0: 0]$. This implies that $c_{11}=c_{22}=0$, i.e. $k=\left(\begin{array}{ccc}0 & c_{12} & 0 \\ c_{21} & 0 & 0 \\ 0 & 0 & 1\end{array}\right)$. However, this contradicts that the defining equation of the curve $C$ has a $Z X^{d-1}$ term but no $Z Y^{d-1}$ term. Therefore, the group Aut $(C)$ is a cyclic group.

We give an example of a smooth plane curve which satisfies the condition of the (1) of Theorem 1.2 but not the condition of the (1) of Theorem 1.1.

Example 3.4 Let $C$ be a smooth plane curve of degree $d:=3(2 k+1)$ defined by

$$
Y X^{d-1}+Y^{d}+Z^{d}+X^{\frac{d-1}{2}} Y^{\frac{d+3}{6}} Z^{\frac{d}{3}}=0
$$


where $k \geq 1$. Let $l:=\frac{d-1}{2} \frac{d}{3}$. The curve $C$ has an automorphism $\sigma$ of order $l$ :

$$
\sigma:=\left(\begin{array}{ccc}
e_{l} & 0 & 0 \\
0 & e_{l}^{1-d} & 0 \\
0 & 0 & 1
\end{array}\right)
$$

By Theorem 3.3, the automorphism group $\operatorname{Aut}(C)$ is a cyclic group. From here, we will show that the order of $\operatorname{Aut}(C)$ is $l$. Let $\varsigma$ be a generator of $\operatorname{Aut}(C)$, and $t:=\operatorname{ord}(\varsigma)$. Since $\sigma$ and $\varsigma$ are commutative, the automorphism $\varsigma$ is defined by a diagonal matrix. Since $\frac{d-1}{2} \frac{d}{3}$ divides $t$, by Theorem 2.12, we get that

$$
\varsigma=\left(\begin{array}{ccc}
e_{t} & 0 & 0 \\
0 & e_{t}^{1-d} & 0 \\
0 & 0 & 1
\end{array}\right)
$$

and $t$ divides $(d-1) d$. Let $u:=\operatorname{gcd}(t, d-1)$ and $v:=\operatorname{gcd}(t, d)$. Then $t=u v$,

$$
\varsigma^{u}=\left(\begin{array}{ccc}
e_{v} & 0 & 0 \\
0 & e_{v} & 0 \\
0 & 0 & 1
\end{array}\right), \text { and } \varsigma^{v}=\left(\begin{array}{ccc}
e_{u} & 0 & 0 \\
0 & 1 & 0 \\
0 & 0 & 1
\end{array}\right) \text {. }
$$

By the defining equation of $C$, we get that $\operatorname{ord}\left(\varsigma^{u}\right)=v$ divides $\frac{d}{3}$, and $\operatorname{ord}\left(\varsigma^{v}\right)=u$ divides $\frac{d-1}{2}$. Therefore, the order of $\operatorname{Aut}(C)$ is $l=\frac{d-1}{2} \frac{d}{3}$.

Next, we will show the (2) of Theorem 1.2.

Theorem 3.5 Let $C$ be a smooth plane curve of degree $d \geq 4$, and Aut $(C)$ be the automorphism group of $C$. We assume that $C$ has an automorphism $\sigma$ such that $\operatorname{ord}(\sigma) \geq 8$ and $\operatorname{gcd}(\operatorname{ord}(\sigma), d) \geq 2$.

1. If $\operatorname{gcd}(\operatorname{ord}(\sigma), d-1) \geq 3$, then $\operatorname{Aut}(C)$ is a cyclic group, or there is a subgroup $H$ of $\operatorname{Aut}(C)$ such that the quotient group $\operatorname{Aut}(C) / H$ is the tetrahedral group $A_{4}$.

2. If $\operatorname{gcd}(\operatorname{ord}(\sigma), d-1) \geq 4$, then $\operatorname{Aut}(C)$ is a cyclic group.

Proof Let $\sigma \in \operatorname{Aut}(C)$ be an automorphism such that $\operatorname{ord}(\sigma) \geq 8, \operatorname{gcd}(\operatorname{ord}(\sigma), d) \geq 2$, and $\operatorname{gcd}(\operatorname{ord}(\sigma), d-$ $1) \geq 3$. By Lemmas 2.3 and 2.6, the pair $(C$, Aut $(C))$ is not the case $(b-i)$ or $(b-i i)$ of Theorem 2.11. Since $\operatorname{ord}(\sigma) \geq 8$, the pair $(C, \operatorname{Aut}(C))$ is not the case $(c)$ of Theorem 2.11. Then we get that the pair $(C, \operatorname{Aut}(C))$ is the case $(a-i)$ or $(a-i i)$ of Theorem 2.11 .

We assume that the pair $(C$, Aut $(C))$ is the case $(a-i i)$ of Theorem 2.11. We assume that the quotient group $\operatorname{Aut}(C) / H$ is a cyclic group. Let $\zeta \in \operatorname{Aut}(C)$ be a generator of the cyclic group $\operatorname{Aut}(C) / H$. Then $\varsigma^{i}=\sigma$ as an element of $\operatorname{Aut}(C) / H$ for some $i$. Since the order of $H$ divides $d, \operatorname{and} \operatorname{gcd}(\operatorname{ord}(\sigma), d-1) \geq 3$, we get that $\operatorname{gcd}(\operatorname{ord}(\varsigma), d-1) \geq 3$. By Theorem 2.12 and Lemma 3.2, by replacing the local coordinate system, $[0: 0: 1] \notin C$, Aut $(C) \subset \operatorname{PBD}(2,1)$, the automorphism $\varsigma$ is defined by a diagonal matrix, and $n(\varsigma) \neq 0$, and hence $C \cap\{[1: 0: 0],[0: 1: 0],[0: 0: 1]\} \neq \emptyset$. Recall that in this local coordinate system, $H=\operatorname{Aut}(C) \cap\left\langle\left(\begin{array}{ccc}e_{d} & 0 & 0 \\ 0 & e_{d} & 0 \\ 0 & 0 & 1\end{array}\right)\right\rangle$. Then $C \cap\{[1: 0: 0],[0: 1: 0],[0: 0: 1]\} \subset \operatorname{Fix}(\tau)$ for any $\tau \in H$. Since $C$ is a smooth curve, $\operatorname{Aut}(C)$ is a cyclic group.

From here, we will show that the quotient group $\operatorname{Aut}(C) / H$ is not one of the groups $D_{m}, \mathcal{S}_{4}$, and $A_{5}$ where $m \leq d-1$. Since the order of $g$ and Lemma 3.2, by replacing the local coordinate system, $[0: 0: 1] \notin C$, and $\operatorname{Aut}(C) \subset \operatorname{PBD}(2,1)$, the pair $(C, \sigma)$ is No. 3 of Table 1 , and $H=\operatorname{Aut}(C) \cap\left\langle\left(\begin{array}{ccc}e_{d} & 0 & 0 \\ 0 & e_{d} & 0 \\ 0 & 0 & 1\end{array}\right)\right\rangle$. Then the automorphsim $\sigma=\left(\begin{array}{ccc}e_{l} & 0 & 0 \\ 0 & e_{l}^{1-d} & 0 \\ 0 & 0 & 1\end{array}\right)$ and $C \cap\{[1: 0: 0],[0: 1: 0],[0: 0: 1]\}=\{[1: 0: 0]\}$ where $l:=\operatorname{ord}(\sigma)$ divides $(d-1) d$. Since ord $(\sigma)$ does not divide $d-1$ and $d, \operatorname{Fix}(\sigma)=\{[1: 0: 0]\}$. We assume that the quotient group $\operatorname{Aut}(C) / H$ is a dihedral group $D_{m}$ where $m \leq d-1$. Let $\varphi: \operatorname{Aut}(C) \rightarrow \operatorname{Aut}(C) / H$ be the quotient morphism. Since $\operatorname{gcd}(\operatorname{ord}(\sigma), d) \geq 2, \operatorname{gcd}(l, d-1) \geq 3$, and the order of the group $H$ divides $d$, we get that the order of an element $\varphi(\sigma)$ of $\operatorname{Aut}(C) / H$ is 3 or more. Then there is an automorphism $k \in \operatorname{Aut}(C)$ 
such that the order of an element $\varphi(\varsigma)$ is 2 and $\varphi(\sigma) \varphi(\varsigma)=\varphi(\varsigma) \varphi(\sigma)^{-1}$. Then for some $\tau \in H$, we get that $\sigma \varsigma=\varsigma \sigma^{-1} \tau$. Since $\tau$ is defined by a diagonal matrix and $\operatorname{Fix}(\sigma)=\{[1: 0: 0]\}$, we get that $\operatorname{Fix}(\sigma) \subset \operatorname{Fix}(\tau)$. By the equation $\sigma \varsigma=\varsigma \sigma^{-1} \tau$, we get that $\sigma(\varsigma([1: 0: 0]))=\varsigma([1: 0: 0])$. Since Fix $(\sigma)=\{[1: 0: 0]\}$, we get that $\varsigma([1: 0: 0])=[1: 0: 0]$, and hence $[1: 0: 0] \in \operatorname{Fix}(\varsigma)$. Since $C$ is a smooth curve and $[1: 0: 0] \in \operatorname{Fix}(\varsigma) \cap \operatorname{Fix}(\sigma)$, the group $\langle\varsigma, \sigma\rangle$ is a cyclic group, and hence $\varsigma \sigma=\sigma \varsigma$. Since the order of an element $\varphi(\sigma)$ is 3 or more, it contradicts the equation $\varphi(\sigma) \varphi(\varsigma)=\varphi(\varsigma) \varphi(\sigma)^{-1}$. Therefore, the quotient group $\operatorname{Aut}(C) / H$ is not a dihedral group $D_{m}$ for $m \leq d-1$.

Since the order of an element of $\mathcal{S}_{4}, A_{4}$, and $A_{5}$ is at most 5 , if $\operatorname{gcd}(l, d-1) \geq 6$, then the quotient group $\operatorname{Aut}(C) / H$ is a cyclic group, and hence $\operatorname{Aut}(C)$ is a cyclic group. Let $s$ be an element of $\mathcal{S}_{4}$, and $l$ be the order of $s$. Then $l$ is 2,3 , or 4 . If $l=3$ or 4 , then there is a subgroup $G_{l}$ of $\mathcal{S}_{4}$ such that $s \in G_{l}$ and $G_{l}$ is the dihedral group of degree $l$. Therefore, if $\operatorname{gcd}(l, d-1) \geq 3$, then the quotient group $\operatorname{Aut}(C) / H$ is not $\mathcal{S}_{4}$. In the same way, we get that if $\operatorname{gcd}(l, d-1) \geq 3$, then the quotient group $\operatorname{Aut}(C) / H$ is not $A_{5}$. In addition, since the order of an element of the group $A_{4}$ is 2 or 3 , if $\operatorname{gcd}(l, d-1) \geq 4$, then the group $\operatorname{Aut}(C)$ is a cyclic group.

We give an example of a smooth plane curve which satisfies the condition of the (2) of Theorem 1.2 but not the condition of the (1) of Theorem 1.1.

Example 3.6 Let $C$ be a smooth plane curve of degree $d:=6(3 k+1)$ defined by

$$
Z X^{d-1}+X Y^{d-1}+Z^{d}+X^{2} Y^{\frac{d-1}{2}} Z^{\frac{d-3}{2}}=0
$$

where $k \geq 1$. Let $l:=3 \frac{d-1}{2}=3^{2}(3 k+1)$. The curve $C$ has an automorphism $\sigma$ of order $l$ :

$$
\sigma:=\left(\begin{array}{ccc}
e_{l}^{1-d} & 0 & 0 \\
0 & e_{l} & 0 \\
0 & 0 & 1
\end{array}\right)
$$

By Theorem 3.5, the automorphism group $\operatorname{Aut}(C)$ is a cyclic group. From here, we will show that the order of $\operatorname{Aut}(C)$ is $l$. Let $\varsigma$ be a generator of $\operatorname{Aut}(C)$, and $t:=\operatorname{ord}(\varsigma)$. Since $\sigma$ and $\varsigma$ are commutative, the automorphism $\varsigma$ is defined by a diagonal matrix. Since $3 \frac{d-1}{2}$ divides $t$, by Theorem 2.12 , we get that

$$
\varsigma=\left(\begin{array}{ccc}
e_{t}^{1-d} & 0 & 0 \\
0 & e_{t} & 0 \\
0 & 0 & 1
\end{array}\right)
$$

and $t$ divides $(d-1)^{2}$. By the defining equation of $C$, we get that ord $(\varsigma)=t$ divides $3 \frac{d-1}{2}$. Therefore, the order of $\operatorname{Aut}(C)$ is $l=3 \frac{d-1}{2}$.

From here, we show the case (3) of Theorem 1.2.

Lemma 3.7 Let $C$ be a smooth plane curve of degree $d \geq 4$, and $\operatorname{Aut}(C)$ be the automorphism group of $C$. If there is an automorphism $\sigma \in \operatorname{Aut}(C)$ such that $\operatorname{gcd}(\operatorname{ord}(\sigma), d-2) \geq 12$, then the pair $(C$, Aut $(C))$ is the case $(a-i)$ or (a-ii) of Theorem 2.11.

Proof Let $\sigma \in \operatorname{Aut}(C)$ be an automorphism such that $\operatorname{gcd}(\operatorname{ord}(\sigma), d-2) \geq 12$. Since $\operatorname{ord}(\sigma) \geq 8$, the pair $(C$, Aut $(C))$ is not the case $(c)$ of Theorem 2.11. Since Lemmas 2.3 and 2.6, the pair $(C$, Aut $(C))$ is neither the case $(b-i)$ nor the case $(b-i i)$ of Theorem 2.11. Therefore, the pair $(C$, Aut $(C))$ is the case $(a-i)$ or $(a-i i)$ of Theorem 2.11.

By Theorem 2.12, if $\operatorname{gcd}(\operatorname{ord}(\sigma), d-2) \geq 12$, then ord $(\sigma)$ divides $(d-2) d$. The following Theorem 3.8 corresponds to the (3) of Theorem 1.2.

Theorem 3.8 Let $C$ be a smooth plane curve of degree $d \geq 4$, and $\operatorname{Aut}(C)$ be the automorphism group of $C$. If there is an automorphism $\sigma \in \operatorname{Aut}(C)$ such that $\operatorname{gcd}(\operatorname{ord}(\sigma), d-2) \geq 12$, then $\operatorname{Aut}(C)$ is a cyclic group, or there is a normal cyclic group $H$ of $\operatorname{Aut}(C)$ such that the quotient group $\operatorname{Aut}(C) / H$ is $D_{m}$, and the order of $H$ divides $d$ where $m$ divides $d-2$. 
Proof Let $\sigma \in \operatorname{Aut}(C)$ be an automorphism such that $\operatorname{gcd}(\operatorname{ord}(\sigma), d-2) \geq 12$. By Lemma 3.7, the pair $(C$, Aut $(C))$ is the case $(a-i)$ or $(a-i i)$ of Theorem 2.11. We assume that the pair $(C$, Aut $(C))$ is the case $(a-i i)$ of Theorem 2.11. Since Lemma 3.2, $\operatorname{ord}(\sigma)$ and Theorem 2.12, by replacing the local coordinate system, $[0: 0$ : $1] \notin C$, and $\operatorname{Aut}(C) \subset \operatorname{PBD}(2,1)$, and the pair $(C, \sigma)$ is No. 7 of Table 1 . Then the automorphism $\sigma$ is defined by a diagonal matrix $\left(\begin{array}{ccc}e_{l} & 0 & 0 \\ 0 & e_{l}^{1-d} & 0 \\ 0 & 0 & 1\end{array}\right)$, and $C \cap\{[1: 0: 0],[0: 1: 0],[0: 0: 1]\}=\{[1: 0: 0],[0: 1: 0]\}$. Let $H=\operatorname{Aut}(C) \cap\left\langle\left(\begin{array}{ccc}e_{d} & 0 & 0 \\ 0 & e_{d} & 0 \\ 0 & 0 & 1\end{array}\right)\right\rangle$. Then the quotient group $\operatorname{Aut}(C) / H$ is one of $\mathcal{G}_{d}$. Let $q: \operatorname{Aut}(C) \rightarrow \operatorname{Aut}(C) / H$ be the quotient morphism. Since $|H|$ divides $d, \operatorname{gcd}(d-2, d) \leq 2$ and $\operatorname{gcd}(\operatorname{ord}(\sigma), d-2) \geq 12$, we get that the order of $q(\sigma)$ is at least 6 as an element of $\operatorname{Aut}(C) / H$. Then, the quotient group $\operatorname{Aut}(C) / H$ is $\mathcal{C} y c_{m}$ or $D_{m}$. Since $C \cap\{[1: 0: 0],[0: 1: 0],[0: 0: 1]\} \neq \emptyset$, if the quotient group $\operatorname{Aut}(C) / H$ is $\mathcal{C} y c_{m}$, then $\operatorname{Aut}(C)$ is a cyclic group.

As be seen from the following two Examples 3.9 and 3.10, either case of Theorem 3.8 is occurred. Therefore, if $\operatorname{ord}(\sigma)$ divides $(d-2) d$, then the structure of $\operatorname{Aut}(C)$ is may not determined by $\operatorname{ord}(\sigma)$.

Example 3.9 Let $C_{1}$ be a smooth plane curve of degree $d:=8 k+6$ defined by

$$
Y X^{d-1}+X Y^{d-1}+Z^{d}+X^{\frac{3 d-2}{8}} Y^{\frac{d+2}{8}} Z^{\frac{d}{2}}=0 .
$$

Let $l:=\left(\frac{d-2}{4}\right)\left(\frac{d}{2}\right)$. The curve $C_{1}$ has an automorphism

$$
\sigma:=\left(\begin{array}{ccc}
e_{l} & 0 & 0 \\
0 & e_{l}^{1-d} & 0 \\
0 & 0 & 1
\end{array}\right)
$$

From here, we will show that the automorphism group $\operatorname{Aut}\left(C_{1}\right)$ is a cyclic group of order $l$. We assume that $\operatorname{Aut}\left(C_{1}\right)$ is not a cyclic group. Since $\operatorname{gcd}(\operatorname{ord}(\sigma), d-2) \geq 12$, by Theorem 3.8, there is a normal cyclic group $H$ such that $H$ is contained in the center of $\operatorname{Aut}\left(C_{1}\right)$, the order of $H$ divides $d$, and the quotient group $\operatorname{Aut}\left(C_{1}\right) / H$ is $D_{m}$ where $m$ divides $d-2$. Since $\sigma^{\frac{d-2}{4}} \in H$, and $\operatorname{Aut}\left(C_{1}\right) / H$ is $D_{m}$, there is an automorphism $\varsigma$ such that $\varsigma \notin H, \varsigma^{2} \in H$, and $\varsigma \sigma \varsigma \sigma \in H$. Recall that since the pair $\left(C_{1}\right.$, Aut $\left.\left(C_{1}\right)\right)$ is the case $(a-i i)$ of Theorem 2.11, there is one invariant point $p \in \mathbb{P}^{2} \backslash C_{1}$ under the action of $\operatorname{Aut}\left(C_{1}\right)$. From the defining matrix of the automorphism $\sigma$, the above-mentioned $p$ is $[0: 0: 1]$. Since $\varsigma([0: 0: 1])=[0: 0: 1]$, the automorphism $\varsigma$ is defined by a matrix of the form

$$
\left(\begin{array}{ccc}
a_{11} & a_{12} & 0 \\
a_{21} & a_{22} & 0 \\
a_{31} & a_{32} & a_{33}
\end{array}\right)
$$

Since $\varsigma \sigma^{\frac{d-2}{4}}=\sigma^{\frac{d-2}{4}} \varsigma$ and the automorphism $\sigma^{\frac{d-2}{4}}$ is defined by a matrix of the form

$$
\left(\begin{array}{lll}
a & 0 & 0 \\
0 & a & 0 \\
0 & 0 & 1
\end{array}\right)
$$

we get that $a_{31}=a_{32}=0$. Since $\varsigma \sigma \varsigma \sigma \in H, \sigma^{2} \notin H$, and $\operatorname{Fix}(\sigma)=\{[1: 0: 0]$, [0:1:0]\}, we get that $a_{11}=a_{22}=0$. This contradicts that $\varsigma$ is an automorphism of $C_{1}$ from the form of the defining equation of $C_{1}$. Therefore, the group $\operatorname{Aut}\left(C_{1}\right)$ is a cyclic group.

Next, we show that the order of $\operatorname{Aut}\left(C_{1}\right)$ is $l=\left(\frac{d-2}{4}\right)\left(\frac{d}{2}\right)$. Let $\tau \in \operatorname{Aut}\left(C_{1}\right)$ be a generator. Since the group Aut $\left(C_{1}\right)$ is a cyclic group, $\tau^{i}=\sigma$ for some $i$. In particular, ord $(\sigma)$ divides ord $(\tau)$. By Theorem 2.12, the pair $\left(C_{1}, \tau\right)$ is No. 7 of Table 1 . Then Fix $(\tau)$ is a set of two points, and there is only one point of $\mathbb{P}^{2} \backslash C_{1}$ that is invariant under the action of $\tau$. Since $\tau^{i}=\sigma$ for some $i$, we get that $\operatorname{Fix}(\tau)=\{[1: 0: 0],[0: 1: 0]\}$, and 
$\tau([0: 0: 1])=[0: 0: 1]$. Then the automorphism $\tau$ is defined by a diagonal matrix. In addition, by the core of the defining equation of $C_{1}$, the automorphism $\tau$ is defined by a matrix of the form

$$
\left(\begin{array}{ccc}
e_{m} & 0 & 0 \\
0 & e_{m}^{1-d} & 0 \\
0 & 0 & 1
\end{array}\right)
$$

where $m:=\operatorname{ord}(\tau)$. Since the defining equation of $C_{1}$ has a term $X^{\frac{3 d-2}{8}} Y^{\frac{d+2}{8}} Z^{\frac{d}{2}}$,

$$
d-1 \equiv \frac{3 d-2}{8}+(1-d) \frac{d+2}{8}(\bmod m) \text {. }
$$

Then

$$
0 \equiv\left(\frac{d-2}{4}\right)\left(\frac{d}{2}\right)(\bmod m)
$$

This implies that $m$ divides $l=\left(\frac{d-2}{4}\right)\left(\frac{d}{2}\right)$. Therefore, Aut $\left(C_{1}\right)$ is a cyclic group of order $l=\left(\frac{d-2}{4}\right)\left(\frac{d}{2}\right)$.

Example 3.10 Let $C_{2}$ be a smooth plane curve of degree $d:=8 k+6$ defined by

$$
Y X^{d-1}+X Y^{d-1}+Z^{d}+X^{\frac{3 d-2}{8}} Y^{\frac{d+2}{8}} Z^{\frac{d}{2}}+X^{\frac{d+2}{8}} Y^{\frac{3 d-2}{8}} Z^{\frac{d}{2}}=0 .
$$

Let $l:=\left(\frac{d-2}{4}\right)\left(\frac{d}{2}\right)$. The curve $C_{2}$ has two automorphism

$$
\sigma:=\left(\begin{array}{ccc}
e_{l} & 0 & 0 \\
0 & e_{l}^{1-d} & 0 \\
0 & 0 & 1
\end{array}\right) \text { and } \varsigma:=\left(\begin{array}{lll}
0 & 1 & 0 \\
1 & 0 & 0 \\
0 & 0 & 1
\end{array}\right) .
$$

As like Example 3.9, we get that the quotient group $\operatorname{Aut}\left(C_{2}\right) /\left\langle\sigma^{\frac{d-2}{4}}\right\rangle$ is $D_{\frac{d-2}{4}}$.

We give a sufficient condition for the pair $(C, \operatorname{Aut}(C))$ to be a descendant of the Klein curve $K_{d}$.

Theorem 3.11 Let $C$ be a smooth plane curve of degree $d \geq 4$, and $\operatorname{Aut}(C)$ be the automorphism group of $C$. If there is an automorphism $\sigma \in \operatorname{Aut}(C)$ such that $\operatorname{ord}(\sigma)$ divides $(d-2)(d-1)+1$, and $\operatorname{ord}(\sigma) \geq 8$, then the pair $(C, \operatorname{Aut}(C))$ is a descendant of the Klein curve $K_{d}$.

Proof Let $\sigma \in \operatorname{Aut}(C)$ be an automorphism such that ord $(\sigma)$ divides $(d-2)(d-1)+1$, and $\operatorname{ord}(\sigma) \geq 8$.

First, we assume that $\operatorname{Aut}(C)$ is a cyclic group. Let $\zeta$ be a generator of the group Aut $(C)$. Then ord( $(\varsigma)$ is a multiple of $\operatorname{ord}(\sigma)$. Since $\operatorname{gcd}((d-2)(d-1)+1, d-2)=1, \operatorname{gcd}((d-2)(d-1)+1, d-1)=1$, $\operatorname{gcd}((d-2)(d-1)+1, d)=1$ or 3 , and Theorem 2.12, we get that the pair $(C, \varsigma)$ is No. 10 of Table 1 . Then the pair $(C, \operatorname{Aut}(C))$ is a descendant of the Klein curve $K_{d}$.

Next, we assume that $\operatorname{Aut}(C)$ is not a cyclic group. Since the group $\operatorname{Aut}(C)$ has an element whose order $l \geq 8$, the pair $(C, \operatorname{Aut}(C))$ is not the case $(c)$ of Theorem 2.11. By Corollary 2.3, the pair $(C$, Aut $(C))$ is not the case $(b-i)$ of Theorem 2.11. We assume that the pair $(C$, Aut $(C))$ is the case $(a-i i)$ of Theorem 2.11. Since Lemma 3.2, by replacing the local coordinate system if necessary, we may assume that $[0: 0: 1] \notin C$, and the automorphism $\sigma$ is defined by a diagonal matrix. Since ord $(\sigma)$ divides $(d-2)(d-1)+1$, and $\operatorname{ord}(\sigma)>3$, by Theorem 2.12, the pair $(C, \sigma)$ is No. 10 of Table 1 , and hence $n(\sigma)=3$. This contradicts that $[0: 0: 1] \notin C$. Therefore, the pair $(C, \operatorname{Aut}(C))$ is not the case $(a-i i)$ of Theorem 2.11. As a result, the pair $(C, \operatorname{Aut}(C))$ is the case $(b-i i)$ of Theorem 2.11, i.e. the pair $(C$, Aut $(C))$ is a descendant of the Klein curve $K_{d}$.

By the following two Examples 3.12 and 3.13 , if ord $(\sigma)$ divides $(d-2)(d-1)+1$, then the structure of $\operatorname{Aut}(C)$ is may not determined by ord $(\sigma)$.

Example 3.12 Let $C_{3}$ be a smooth plane curve of degree $d:=7 k+6$ defined by

$$
Z X^{d-1}+X Y^{d-1}+Y Z^{d-1}+X^{k+1} Y^{2 k+2} Z^{4 k+3}=0 .
$$

Let $l:=\frac{(d-2)(d-1)+1}{7}=7 k^{2}+9 k+3$. The curve $C_{3}$ has an automorphism

$$
\sigma:=\left(\begin{array}{ccc}
e_{l} & 0 & 0 \\
0 & e_{l}^{d-1} & 0 \\
0 & 0 & 1
\end{array}\right) .
$$


From here, we show that the automorphism group $\operatorname{Aut}\left(C_{3}\right)$ is a cyclic group of order $l$. We assume that $\operatorname{Aut}\left(C_{3}\right)$ is not a cyclic group. Since ord $(\sigma)$ divides $(d-2)(d-1)+1, \operatorname{ord}(\sigma) \geq 8$, and Theorem 3.11, there is an automorphism $\varsigma$ such that $\operatorname{ord}(\varsigma)=3$, and $\langle\varsigma\rangle \cap\langle\sigma\rangle=\left\{\operatorname{id}_{C_{3}}\right\}$. Let $\tau \in \operatorname{Aut}\left(C_{3}\right)$ such that ord $(\tau)$ is the largest order among the automorphisms of $\operatorname{Aut}\left(C_{3}\right)$. Since ord $(\sigma) \neq 3$, and Theorem 2.5, $\varsigma^{i}=\sigma$ for some $i$. Then $\operatorname{ord}(\sigma)$ divides $\operatorname{ord}(\tau)$. By Theorem 2.12, the pair $(C, h)$ is No. 10 of Table 1. Then Fix $(\tau)$ is a set of three points. Since $\tau^{i}=\sigma$ for some $i, \operatorname{Fix}(\tau)=\operatorname{Fix}(\sigma)=\{[1: 0: 0],[0: 1: 0],[0: 0: 1]\}$. Since $\langle\tau\rangle$ is a normal group of $\operatorname{Aut}\left(C_{3}\right)$, we may assume that the automorphism $\varsigma$ is defined by a matrix of the form

$$
\left(\begin{array}{ccc}
0 & a_{12} & 0 \\
0 & 0 & a_{23} \\
a_{31} & 0 & 0
\end{array}\right)
$$

This contradicts that the form of the defining equation of $C_{3}$. Therefore, $\operatorname{Aut}\left(C_{3}\right)$ is a cyclic group.

Next, we show that the order of the group Aut $\left(C_{3}\right)$ is $l=\frac{(d-2)(d-1)+1}{7}=7 k^{2}+9 k+3$. Let $\tau \in \operatorname{Aut}\left(C_{3}\right)$ be a generator. Since $\operatorname{Aut}\left(C_{3}\right)$ is a cyclic group, $\tau^{i}=$ for some $i$. In particular, ord $(\sigma)$ divides ord $(\tau)$. By Theorem 2.12, the pair $\left(C_{3}, \tau\right)$ is No. 10 of Table 1. Then Fix $(\tau)$ is a set of three points. Since $\tau^{i}=\sigma$ for some $i$, we get that $\operatorname{Fix}(\tau)=\{[1: 0: 0],[0: 1: 0],[0: 0: 1]\}$. Then the automorphism $\tau$ is defined by a diagonal matrix. In addition, by the core of the defining equation of $C_{3}$, the automorphism $\tau$ is defined by a matrix of the form

$$
\left(\begin{array}{ccc}
e_{m} & 0 & 0 \\
0 & e_{m}^{d-1} & 0 \\
0 & 0 & 1
\end{array}\right)
$$

where $m:=\operatorname{ord}(\tau)$. Since the defining equation of $C_{3}$ has a term $X^{k+1} Y^{2 k+2} Z^{4 k+3}$,

$$
d-1 \equiv(k+1)+(d-1)(2 k+2)(\bmod m) .
$$

Then

$$
0 \equiv 2\left(7 k^{2}+9 k+3\right)(\bmod m) .
$$

This implies that $m$ divides $\frac{2}{7}((d-2)(d-1)+1)$. Therefore, Aut $\left(C_{3}\right)$ is a cyclic group of order $l=$ $\frac{1}{7}((d-2)(d-1)+1)$.

Example 3.13 Let $C_{4}$ be a smooth plane curve of degree $d:=7 k+6$ defined by

$$
Z X^{d-1}+X Y^{d-1}+Y Z^{d-1}+X^{k+1} Y^{2 k+2} Z^{4 k+3}+X^{4 k+3} Y^{k+1} Z^{2 k+2}+X^{2 k+2} Y^{4 k+3} Z^{k+1}=0 .
$$

Let $l:=\frac{(d-2)(d-1)+1}{7}=7 k^{2}+9 k+3$. The curve $C_{4}$ has two automorphism

$$
\sigma:=\left(\begin{array}{ccc}
e_{l} & 0 & 0 \\
0 & e_{l}^{d-1} & 0 \\
0 & 0 & 1
\end{array}\right) \text { and } \tau:=\left(\begin{array}{lll}
0 & 1 & 0 \\
0 & 0 & 1 \\
1 & 0 & 0
\end{array}\right) .
$$

Then $\operatorname{Aut}\left(C_{4}\right)$ is not a cyclic group, and the quotient group $\operatorname{Aut}\left(C_{4}\right) /\langle g\rangle$ is $\mathcal{C} y c_{3}$.

Lastly, we will characterize the Fermat curve $F_{d}$ using an automorphism of order $2 d$ and an automorphism of order 3 for a smooth plane curve of degree $d$.

Theorem 3.14 Let $C$ be a smooth plane curve whose degree $d \geq 4$, and $\operatorname{Aut}(C)$ be the automorphism group of $C$. If $C$ has two automorphism $\sigma$ and $\varsigma$ such that $\operatorname{ord}(\sigma)=2 d, \operatorname{ord}(\varsigma)=3$, and $\operatorname{ord}\left(\varsigma \sigma^{2}\right)=3$, then $C$ is projectively equivalent to the Fermat curve $F_{d}$.

Proof Let $\sigma \in \operatorname{Aut}(C)$ be an automorphism such that $\operatorname{ord}(\sigma)=2 d$. By Theorem 2.20, $C$ has an outer Galois point. Let $\delta^{\prime}(C)$ be the number of outer Galois points of $C$. By Theorem 2.19, $\delta^{\prime}(C)=1$ or 3, and if $\delta^{\prime}(C)=3$, then $C$ is projectively equivalent to the Fermat curve $F_{d}$. We assume that $\delta^{\prime}(C)=1$. By Theorem 2.19, the pair $(C$, Aut $(C))$ is the case $(a-i i)$. Since the order of $\sigma$ and Lemma 3.2, by replacing the local coordinate system, $[0: 0: 1] \notin C$, and $\operatorname{Aut}(C) \subset \operatorname{PBD}(2,1)$, and the pair $(C, \sigma)$ is No. 3 of Table 1 . Then the automorphism $\sigma$ 
is defined by a diagonal matrix $\left(\begin{array}{ccc}e_{2 d} & 0 & 0 \\ 0 & e_{2 d}^{1-d} & 0 \\ 0 & 0 & 1\end{array}\right)$. Then the automorphism $\sigma^{2}$ is defined by a matrix $\left(\begin{array}{ccc}e_{d} & 0 & 0 \\ 0 & e_{d} & 0 \\ 0 & 0 & 1\end{array}\right)$. Since $\operatorname{Aut}(C) \subset \operatorname{PBD}(2,1)$, the automorphism $\varsigma$ is defined by a matrix of the form $\left(\begin{array}{lll}a & b & 0 \\ c & d & 0 \\ 0 & 0 & 1\end{array}\right)$. This contradicts that $\operatorname{ord}(\sigma)=2 d, \operatorname{ord}(\varsigma)=3$, and $\operatorname{ord}\left(\varsigma \sigma^{2}\right)=3$. Therefore, $\delta^{\prime}(C)=3$, and hence the curve $C$ is projectively equivalent to the Fermat curve $F_{d}$.

By Theorem 2.2, $\left.\sigma:=\left(\begin{array}{lll}0 & e_{d} & 0 \\ 1 & 0 & 0 \\ 0 & 0 & 1\end{array}\right)\right\rangle$ and $\left.\varsigma:=\left(\begin{array}{lll}0 & 1 & 0 \\ 0 & 0 & 1 \\ 1 & 0 & 0\end{array}\right)\right\rangle$ are automorphisms of the Fermat curve $F_{d}$ such that $\operatorname{ord}(\sigma)=2 d, \operatorname{ord}(\varsigma)=3$, and $\operatorname{ord}\left(\varsigma \sigma^{2}\right)=3$.

Open Access This article is licensed under a Creative Commons Attribution 4.0 International License, which permits use, sharing, adaptation, distribution and reproduction in any medium or format, as long as you give appropriate credit to the original author(s) and the source, provide a link to the Creative Commons licence, and indicate if changes were made. The images or other third party material in this article are included in the article's Creative Commons licence, unless indicated otherwise in a credit line to the material. If material is not included in the article's Creative Commons licence and your intended use is not permitted by statutory regulation or exceeds the permitted use, you will need to obtain permission directly from the copyright holder. To view a copy of this licence, visit http://creativecommons.org/licenses/by/4.0/.

\section{References}

1. Bars, F.: On the automorphisms groups of genus 3 curves. Surv. Math. Sci. 2(2), 83-124 (2012)

2. Badr, E.; Bars, F.: Automorphism groups of non-singular plane curves of degree 5. Commun. Algebra 44, 4327-4340 (2016)

3. Badr, E.; Bars, F.: Non-singular plane curves with an element of "large" order in its automorphism group. Int. J. Algebra Comput. 26, 399-434 (2016)

4. Blichfeldt, H.: Finite Collineation Groups: With an Introduction to the Theory of Groups of Operators and Substitution Groups. Univ. of Chicago Press, Chicago (1917)

5. Fukasawa, S.; Miura, K.; Takahashi, T.: Quasi-Galois points, I: automorphism groups of plane curves. Tohoku Math. J. (2) 71(4), 487-494 (2019)

6. Harui, T.: Automorphism groups of smooth plane curves. Kodai Math. J. 42(2), 308-331 (2019)

7. Harui, T.; Miura, K.; Ohbuchi, A.: Automorphism group of plane curve computed by Galois points, II. Proc. Jpn. Acad. Ser. A Math. Sci. 94(6), 59-63 (2018)

8. Harui, T.; Kato, T.; Komeda, J.; Ohbuchi, A.: Quotient curves of smooth plane curves with automorphisms. Kodai Math. J. 33(1), 164-172 (2010)

9. Hayashi, T.: Smooth plane curves with freely acting finite groups, Vietnam. J. Math. https://doi.org/10.1007/s10013-02000398-z

10. Hayashi, T.: Linear automorphisms of hypersurfaces giving Galois points. arXiv:2101.04797

11. Henn, P.: Die Automorphismengruppen dar algebraischen Functionenkorper vom Geschlecht 3. Inagural-dissertation, Heidelberg (1976).

12. Komeda, J.; Takahashi, T.: Relating Galois points to weak Galois Weierstrass points through double coverings of curves. J. Korean Math. Soc. 54(1), 69-86 (2017)

13. Komeda, J.; Takahashi, T.: Galois Weierstrass points whose Weierstrass semigroups are generated by two elements. arXiv: 1703.09416

14. Kuribayashi, A.; Komiya, K.: On Weierstrass points of non-hyperelliptic compact Riemann surfaces of genus three. Hiroshima Math. J. 7, 743-786 (1977)

15. Miura, K.; Ohbuchi, A.: Automorphism group of plane curve computed by Galois points. Beitr. Algebra Geom. 56(2), 695-702 (2015)

16. Miura, K.; Yoshihara, H.: Field theory for function fields of plane quartic curves. J. Algebra 226, $283-294$ (2000)

17. Namba, M.: Geometry of Projective Algebraic Curves. Marcel Dekker, New York (1984)

18. Pambianco, F.: Characterization of the Fermat curve as the most symmetric nonsingular algebraic plane curve. Math. Z 277, 975-993 (2014)

19. Tzermias, P.: The group of automorphisms of the Fermat curve. J. Num. Theory 53(1), 173-178 (1995)

20. Yoshihara, H.: Function field theory of plane curves by dual curves. J. Algebra 239, 340-355 (2001)

Publisher's Note Springer Nature remains neutral with regard to jurisdictional claims in published maps and institutional affiliations. 